\title{
Prevalence and Associated Factors of Sexually Transmitted Infections Based on the Syndromic Approach among HIV Patients in ART Clinic; Ayder Referral Hospital, Northern Ethiopia
}

\author{
Abraha Gebrelibanos Kahsay ${ }^{1}$, Frehiwot Daba ${ }^{2}$, Abraham Getachew Kelbore ${ }^{1, *}$, \\ Sefonias Getachew ${ }^{3}$
}

${ }^{1}$ Postgraduate in Tropical Dermatology, Mekelle University, Mekelle, North Ethiopia

${ }^{2}$ Dermatovenereology Department head, Mekelle University, Mekelle, North Ethiopia

${ }^{3}$ Addis Ababa University, school of Public Health, Addis Ababa, Ethiopia

\section{Email address:}

abrahaglibanos@gmail.com (A. G. Kahsay),Firemed@yahoo.com (F. Daba), kelbore2005@gmail.com (A. G. Kelbore), safoget@yahoo.com (Sefonias G.)

\section{To cite this article:}

Abraha Gebrelibanos Kahsay, Frehiwot Daba, Abraham Getachew Kelbore, Sefonias Getachew. Prevalence and Associated Factors of Sexually Transmitted Infections Based on the Syndromic Approach among HIV Patients in ART Clinic; Ayder Referral Hospital, Northern Ethiopia. Clinical Medicine Research. Vol. 4, No. 5, 2015, pp. 132-138. doi: 10.11648/j.cmr.20150405.12

\begin{abstract}
Background: Sexually transmitted Infections represent a large burden of disease worldwide with an annual incidence of about 333 million cases. In Ethiopia, studies on Sexually Transmitted Infections (STIs) among HIV patients are very few; therefore, conducting research on STIs in general and among HIV patients in particular is an important input to design policy and strategy aimed at preventing and controlling the infections. Objective: To determine the prevalence and associated factors of sexually transmitted infections among HIV patients in ART clinic; Ayder referral hospital, Tigray, Ethiopia. Methods: Institution based cross sectional study design was conducted among 353 HIV patients in Ayder hospital, ART clinic from July to September 2014. A systematic random sampling technique was used to identify study subjects. Data were collected using structured questionnaire and entered into and analyzed using SPSS 20 for windows. Descriptive analyses were used to estimate the prevalence of STIs and selected characteristics of patients. The effects of predictors on having STIs were analyzed using logistic regression and their effects were depicted using OR adjusted for confounding. P-value less than 0.05 were considered as statistical significant for all tests. Result;: Among total respondents; 150 (42.5\%) were male and 203 (57.5\%) female HIV patients .The prevalence of sexually transmitted infections based on the syndromic approach was $8.5 \%$ with specific prevalence of (4.6\%) urethral discharge syndrome, $(2.8 \%)$ genital ulcer syndrome, none of them scrotal swelling syndrome, $(2.5 \%)$ lower abdominal pain syndrome, $(0.3 \%)$ inguinal bubo and $(5.5 \%)$ had vaginal discharge syndrome. 8 patients $(26.7 \%)$ had recurrent disease and 28 patients $(93.3 \%)$ treated without their partners. After multivariable logistic regression analysis, age $(\mathrm{AOR}=11.3(95 \% \mathrm{CI}$ : $1.1-116.5)$, marital status $(\mathrm{AOR}=0.031$ (95\%CI: 0.001-0.93) and having new sexual partner within the last three months $(A O R=152.7$ (95\%CI: 3.7-6274) were significantly associated with STI syndromes p-value $<0.05$. Conclusion and recommendations: The prevalence of sexually transmitted infections based on the syndromic approach among HIV patients was $8.5 \%$ and the factors associated with STIs among HIV patients were: age, marital status and having new sexual partner within the last three months. Moreover, further studies to explore the predictor variables are highly recommended.
\end{abstract}

Keywords: Prevalence, Sexually Transmitted Infections, Syndrome

\section{Introduction}

Sexually transmitted infections (STIs) are a variety of clinical syndromes caused by pathogens that can be acquired and transmitted through sexual activity which include not only the common classical STIs but also about 20 infections often referred to as "second generation" sexually transmitted infections caused by bacterial, viral, parasitic, Protozoa and 
fungal agents and can be recognized as ulcerative and non-ulcerative or can be classified as curable and incurable. The common curable STIs are gonorrhea, Chlamydia infection, syphilis, chancroid, trichomoniasis, and lymph granuloma venereum and Donovan's. The STIs that are preventable but not curable are the viral STIs which includes human immunodeficiency virus (HIV), Human papilloma virus, hepatitis B virus, and herpes simplex virus [1].

In late 1999 the World Health Organization estimated that globally as many as 340 million new cases of curable STIs (gonorrhea, chlamydial infection, syphilis and trichomoniasis) occurred in 1999, of which 151 million occur in South and South East Asia and 69 million in Sub-Saharan African countries (WHO, 2001. Thus, on average, an estimated 931,500 people are infected every day with STIs. WHO produces these global STI estimates every five years [2].

STIs are a major public health problem in all countries, especially in developing countries, where access to adequate diagnostic and treatment facilities is very limited or non-existent. According to Egyptian, Greek, Roman and Arab writings and drawings, gonorrhea may have been endemic in Africa for more than 2,000 years. Less is known about syphilis, which may have been present in East Africa before the 16th century. The early history of both infections in Ethiopia is obscure, and there is no strong evidence that they spread from the northern highlands to the south as suggested by Guthe [3].

A high prevalence of STIs among prostitutes was reported first by Italian military physicians, who staffed numerous military STI clinics and administered penicillin therapy to large numbers of Italian troops. The many resources devoted by the Italian military to STIs and the numerous publications of case reports and chemotherapy campaigns by military physicians in Italian journals [4].

The ecology of STIs in Ethiopia has become more complex in recent years and reveals many characteristics of risk behavior were Prostitution and increasing poverty resulting postponement of marriage, urbanization, sociocultural change which resulting in sexual behavior, and increasing unemployment and streetism of youth have contributed to earlier and often unprotected sex [5].The prevalence of HIV increases among pregnant mothers who had no partner, this was may be mothers who had no partner practicing unsafe sex, have many partner or may not encourage to use condom their sexual partner [6].

STIs can cause morbidity and increase risk of HIV acquisition and transmission [7]. Due to this, the US Centers for Diseases Control and Prevention (CDC) recommends screening of persons living with HIV/AIDS for STIs, including syphilis, gonorrhea, chlamydia, and (for women) trichomoniasis. Screening for those infections should occur at the first visit and should be repeated periodically [8].

In resource-limited settings, the WHO recommends that health-care providers obtain a thorough STI-related history from all newly diagnosed PLHA; conduct a physical examination to check for STIs; and perform serologic screening for syphilis and (for women) screening for gonorrhea and chlamydia. Accordingly recommends syndromic management of patients with STI symptoms or signs [9].

Diagnosis of presumed STIs has traditionally been based on either clinical diagnosis, which is often inaccurate and incomplete, or laboratory diagnosis, which is complex, very expensive, and may delay treatment. As early as the 1970s, public health physicians, those working in Africa, became interested in testing simple clinical tools for controlling and treating STIs [10].This resulted in the design and promotion of "syndromic management" guidelines for STIs by the World Health Organization in 1991[11].

The syndromic approach does not require identification of the underlying etiology. Instead, it is based on the identification of a syndrome that is, a group of symptoms and easily recognized signs associated with a number of well-defined etiologies. Treatment is provided for the majority of the organisms locally responsible for the syndrome. It rapidly became clear that the syndromic approach offered enormous advantages compared to the traditional approach, although more evidence was needed to rationalize and convince policy makers [12].

\subsection{STIs and HIV Infection}

Both ulcerative and non-ulcerative forms of STIs enhance HIV transmission and that both infections share similar epidemiologic determinants [13]. In India hospital based study shows that syphilis $21.5 \%$ seropositive patients were HIV infected.[14] However patients with HIV and STIs has been found to be associated with the failure of syndromic treatments like herpes cased genital ulcer, genital discharge, genital warts, bacterial vaginosis being HIV positive significantly associated with treatment failure $[15,16]$.

\subsection{Prevention and Control}

On the basis of these various recent developments in STI research, the Ethiopian Ministry of Health now recognizes effective prevention and control of other STIs as (a) a high priority for promoting reproductive health, especially among women; (b) an important approach to preventing cancer and neurologic disease; (c) a very cost- effective approach to preventing neonatal morbidity; and (d) its linkage with HIV/AIDS. Nonetheless, public awareness of the spectrum of adverse health consequences of STIs has been slow to develop, partly because the link between infection and consequences is obscured by the frequently subclinical nature of early infection, especially in women. Other reasons include the long delay until recognition of adverse reproductive health consequences or the occurrence of malignancy or neurologic squeal. Finally, there has not been a major effort to educate the public on the mode of transmission, potentially serious effects of STIs and its linkage with HIV/AIDS [17].

Among women aged 15-44 years both Ulcerative and non-ulcerative STIs have emerged as important public health concerns with increasing gynecological morbidity and 
mortality in women. It is estimated that genital tract infections are the second most frequent cause of healthy life lost after maternal mortality and morbidity [18]. Disproportionately, it affects the health and social wellbeing of women by producing significant impact on their reproductive potential. There is limited information on the incidence and prevalence of STIs in Ethiopia. Due to these reasons; the major contributing factor is that most people with STIs do not seek treatment at public health facilities as they will have minor or no symptoms. They usually tend to take self-prescribed drugs or go to private pharmacies to buy treatment without consulting trained health workers [19].

In Ethiopia, relatively few epidemiological surveys had been carried out on the prevalence and incidence of STIs. However, the problem of STIs in Ethiopia is generally believed to be similar to other developing countries. The Integrated Disease Surveillance Team of the Ministry of Health compiled 58,623 and 27,947 STI cases in 2002 and 2003 respectively using routine quarterly reports [20].

In an STI survey conducted in Ethiopian (65.9\%) presented with vaginal discharge, (25.1\%) with urethral discharge, and (17.6\%) with genital ulcer. N. gonorrhea was the leading pathogen that caused urethral discharge in males as compared to females with vaginal discharge and bacterial vaginosis was the common cause of vaginal discharge in females. Syphilis $(24.0 \%)$ was the second leading cause of genital ulcer in males as compared to females [16].As to our knowledge, there paucity of studies conducted to show the magnitude of STIs among HIV patients, in Northern Ethiopia Therefore this study aimed to determine the magnitude of STIs and associated factors among patients visiting ART clinic in Mekelle town, northern Ethiopia.

\section{Methodology}

\subsection{Study Area and Period}

This study was conducted in Ayder Referral hospital, ART clinic which is found in Mekelle Town; lies $780 \mathrm{~km}$ north of Addis Ababa. The clinic contains four outpatient departments and four health care providers (1 in each OPD), 2 data clerks and two porters. The clinic provides, ART, PRE-ART, VCT and FP services for 993 HIV patients on ART and 290 Pre -ART since January 2001.

The study was conducted from July to September 2014.

\subsection{Study Design, Study Population}

Institution based Cross-sectional study design were used and those all HIV positive patients linked to ART clinic at Ayder referral hospital who were available during the study period and who fulfilled the eligibility criteria of the study were involved.

All patients who were linked to ART clinic, those who agreed to participate in the study by signing an informed consent and Adult patients $>14$ years were included. Patients who had psychiatric illness and children

\subsection{Sample Size Determination and Sampling Techniques}

\subsubsection{Sample Size Determination}

The sample size was determined by using single population proportion formula with confidence interval $95 \%$ and $5 \%$ margin of error by taking prevalence of the case $(35.8 \%)$, from a study conducted at Gondar town clinic, northwest Ethiopia[30] and taking the non-respondent rate of $10 \%$ the final sample size becomes 353 .

\subsubsection{Sampling Technique}

Systematic sampling technique was used to identify the study subjectsand a 764 HIV patients were listed according to their follow up visits in the registration book during the study period and the sampling interval was calculated by using a formula, $\mathrm{k}=\mathrm{N} / \mathrm{n}$ which was, $\mathrm{K}=2$ and the initial sample was selected using lottery method. Then, everysampling interval, $\mathrm{K}=2$ patients were taken for the study.

\subsection{Study Variables}

\subsubsection{Dependent Variable}

Sexually transmitted infections according to syndromic approach

\subsubsection{Independent Variable}

- Sociodemographic factors: -Sex, Age, Educational status, Marital status, Religion, Residence,Occupation and income source.

- Personal habit and practice factors:- New sexual partner, number of sexual partners, condom use, Partner HIV sero-status, use of alcohol and chew khat .

- Disease related clinical factors: - sign and symptoms of syndromic STI's, CD+4 cell count, WHO HIV clinical staging and partner treatment.

\subsection{Data Collection Techniques}

A structure interview questioner was used in the local language once translated from the English version. Additional data was reviewed from clinical examinationandcard of the patients. The interview and clinical examination was conducted among study participants by trained ART clinic clinicians and post graduate dermatology student during ART clinic visits.

\subsection{Quality controland Data Analysis}

Quality control measures was undertaken to increase the reliability of the final out comes. Primarily; the questionnaire was checked by the investigator for its appropriateness such as typing error, missing questions, and inappropriate translations. Health workers (data collectors) were given detailed explanation on the questionnaire to improve their efficiency in collecting unbiased and consistent data. Besides this, the principal investigator was carefully entered and thoroughly cleaned the data before the commencements of the analysis. Finally, the principal investigator was reviewed all filled questionnaires 
A descriptive analysis using Proportion and frequency, mean, standard deviation, wereused.Bivariate logistic regression was applied to see the association between each independent variable with dependent variable and multiple logistic regression model was used to identify independent predictors. Variable found to be significant at $P<0.05$ in the Bivariate analysis were entered to multiple logistic regression. We used the enter approach in for inclusion into the multivariate model while theHosmer-Lemeshow statistic was used for model diagnostics. Statistical significance was declared at Pvalue $<0.05$ and the entered and analysis of the data was performed using SPSS version 20 statistical software package.

\subsection{Ethical Consideration}

Ethical clearance was obtained from the Ethical review committee of the College of health science of Mekelle University. Accordingly, prior to data collection, the medical director of Ayder referral hospital was informed on the general overview of the study and permission was asked as per the recommendation letter from the department. Then, eligible study subjects were informed about the aim of the study and asked for their written consent, and then data was taken from willing subjects.

\subsection{Operational Definition}

Syndromic approach: is identification of consistent groups of symptoms and easily recognized signs (syndromes), and the provision of treatment that will deal with the majority of or the most serious, organisms responsible for producing a syndrome [32].

\section{Results}

\subsection{Socio Demographic Characteristics of Study Subjects}

A total of $353 \mathrm{HIV}$ patients participated in the study making the response rate of $100 \%$. The mean age (standard deviation) of respondents was $35.37 \pm$ (9.68) years with minimum and maximum age of 17 and 78 years, respectively. The most common age groups respondents were 35 years and above $(185 / 52.4 \%)$. Most of study participants were females $(57.5 \%)$ and males (42.5\%) while all most all of the respondents were Orthodox Christians (334/94.6\%) and the rest (19/5.4\%) were Muslims and Protestants. Only 72(20.4\%) of the respondents were illiterate while others complete their primary, secondary and above Diploma and above 160 $(45.3 \%), 80(22.7 \%)$ and $41(11.6 \%)$ respectively. With regard to their marital status, $33.1 \%$ were married, $28.6 \%$ were single and the rest $31.2 \%$ and $7.1 \%$ were divorced and widowed respectively. Most of them participants were urban residents $91.5 \%$

\subsection{Personal Habit and Practice of Study Subjects}

Thirty one respondents $(8.8 \%)$ had new sexual partner within the last three months, Of them 18 respondents $(58.1 \%)$ had one new sexual partner and the rest $41.9 \%$ had more than one new sexual partners and $68.3 \%$ of the participants were used condom regularly during sexual intercourse. Most of them know his or her partner HIV status and the result were $98.2 \%$ positive. Majority of respondents use alcohol type is tella and they didn't chew khat at all.

\subsection{Disease Related Clinical Profiles of Study Subjects}

A 150 male and 203 female patients were enrolled in the study, of them 7 patients (4.6\%) had urethral discharge syndrome, 10 patients $(2.8 \%)$ genital ulcer syndrome, none of them had scrotal swelling syndrome, 5 patients $(2.5 \%)$ had lower abdominal pain syndrome, one patient $(0.3 \%)$ inguinal bubo and 11 patients(5.5\%) had vaginal discharge syndrome.The diagnosis for the syndromes was according to the WHO flow chart for diagnosing sexually transmitted infections based on syndromic approach. 22 patients $(73.3 \%)$ acquire the disease for the first time and the rest 8 patients (26.7\%) had recurrent disease. Only 2 patients $(6.7 \%)$ treated with their partners and the rest 28 patients $(93.3 \%)$ treated without their partners. The overall prevalence of sexually transmitted infections based on the syndromic approach was $8.5 \%$ (30 patients out of 353 study subjects had one or two STI syndromes. $336(95.2 \%)$ of the study subjects were on HAART [Table 1].

Table 1. Frequency distribution of clinical profiles of HIV patients in Ayder hospital ART clinic September 2014/n=353.

\begin{tabular}{|c|c|c|c|}
\hline Variable & Category & Frequency & Percent \\
\hline \multirow{2}{*}{$\begin{array}{l}\text { Urethral discharge } \\
\text { syndrome }\end{array}$} & Yes & 7 & 4.6 \\
\hline & No & 146 & 95.4 \\
\hline \multirow{2}{*}{$\begin{array}{l}\text { Genital ulcer } \\
\text { syndrome }\end{array}$} & Yes & 10 & 2.8 \\
\hline & No & 343 & 97.2 \\
\hline \multirow{2}{*}{$\begin{array}{l}\text { Scrotal swelling } \\
\text { syndrome }\end{array}$} & Yes & 0 & 0 \\
\hline & No & 153 & 100 \\
\hline \multirow{2}{*}{$\begin{array}{l}\text { Lower abdominal } \\
\text { pain syndrome }\end{array}$} & Yes & 5 & 2.5 \\
\hline & No & 195 & 97.5 \\
\hline \multirow{2}{*}{$\begin{array}{l}\text { Inguinal bubo } \\
\text { syndrome }\end{array}$} & Yes & 1 & 0.3 \\
\hline & No & 352 & 99.7 \\
\hline \multirow{2}{*}{$\begin{array}{l}\text { Vaginal discharge } \\
\text { syndrome }\end{array}$} & Yes & 11 & 5.5 \\
\hline & No & 189 & 94.5 \\
\hline \multirow{2}{*}{ Episode } & First & 22 & 73.3 \\
\hline & Recurrent & 8 & 26.7 \\
\hline \multirow{2}{*}{ Partner treatment } & Yes & 2 & 6.7 \\
\hline & No & 28 & 93.3 \\
\hline \multirow{2}{*}{ ART status } & On ART & 336 & 95.2 \\
\hline & Pre ART & 17 & 4.8 \\
\hline \multirow{4}{*}{ CD4 count } & $0-200$ & 92, & 21.1 \\
\hline & $201-350$ & 104 & 29.5 \\
\hline & $351-450$ & 51106 & 14.4 \\
\hline & $>450$ & & 30 \\
\hline \multirow{2}{*}{$\begin{array}{l}\text { WHO clinical } \\
\text { stage }\end{array}$} & I, II, III, IV & $13,12,10,8$ & $3.7,3.4,2.8,2.3$ \\
\hline & $\begin{array}{l}\mathrm{T}-1, \mathrm{~T}-2, \mathrm{~T}-3, \\
\mathrm{~T}-4\end{array}$ & $294,4,2,10$ & $83.3,1.1,0.6,2.8$ \\
\hline \multirow{2}{*}{$\begin{array}{l}\text { STIs based on } \\
\text { syndromes }\end{array}$} & Yes & 30 & 8.5 \\
\hline & No & 323 & 91.5 \\
\hline
\end{tabular}

\subsection{Multivariate Analysis}

Comparison of selected variables that were statistically significant with sexually transmitted infections on crude analysis were adjusted using enter method multivariate 
logistic analysis model. The result of multivariate analysis revealed that were age, marital status and having new partner were significantly associated.

The odds of having STIs were 11.4 times higher among HIV patients with the age range between 15-34yrs, when compared with the age group above or equal to $35 \mathrm{yrs}$. $[\mathrm{AOR}=11.39(95 \% \mathrm{CI}=1.1,116.5)]$. The odds of having STIs among married HIV patients were $96.9 \%$ at reduced risk, when compared with single HIV patients $[\mathrm{AOR}=0.031(95 \% \mathrm{CI}=0.001,0.934)]$. And the odds of having STIs were 152 times higher among HIV patients having new sexual partner within the last three months than the odds of STIs without new sexual partner $[\mathrm{AOR}=152(95 \% \mathrm{CI}=3.7,6224)]$. [Table 2]

Table 2. Multivariate analysis Associated factors on sexually transmitted infections based on syndromic approach among HIV patients, Ayder hospital ART clinic / September, 2014/n=353.

\begin{tabular}{|c|c|c|c|c|}
\hline Variable & Syndromic STIs & & & \\
\hline Age group & No & Yes & $\operatorname{COR}(95 \% \mathrm{CI})$ & $\mathrm{AOR}(95 \% \mathrm{CI})$ \\
\hline $15-34$ & $147(87.5 \%)$ & $21(12.5 \%)$ & $2.794(1.242,6.286)$ & $11.3(1.1,116.5)^{*}$ \\
\hline$\geq 35$ & $176(95.1 \%)$ & $9(4.9 \%)$ & 1 & 1 \\
\hline \multicolumn{5}{|l|}{ Educational status } \\
\hline Illiterate & $67(93.1 \%)$ & $5(6.9 \%)$ & $0.49(0.171,1.399)$ & $2.26(0.156,32.767)$ \\
\hline Grade $1-8$ & $151(94.4 \%)$ & $9(5.6 \%)$ & $0.391(0.167,0.919)$ & $0.455(0.064,3.261)$ \\
\hline \multicolumn{5}{|l|}{ Marital status } \\
\hline Single & $84(83.2 \%)$ & $17(16.8 \%)$ & 1 & 1 \\
\hline Married & $113(96.6 \%)$ & $4(3.4 \%)$ & $0.175(0.057,0.539)$ & $0.031(0.001,0.93)^{*}$ \\
\hline Divorced and widowed & $126(93.3 \%)$ & $9(6.7 \%)$ & $0.353(0.15,0.829)$ & $0.303(0.018,5.227)$ \\
\hline \multicolumn{5}{|l|}{ New sexual partner } \\
\hline Yes & $9(29 \%)$ & $22(71 \%)$ & $95.9(33.7,273.04)$ & $152.71(3.7,6274 .)^{*}$ \\
\hline No & $314(97.5 \%)$ & $8(2.5 \%)$ & 1 & 1 \\
\hline Yes & $44(75.9 \%)$ & $14(24.1 \%)$ & $2.355(0.964,5.752)$ & $0.106(0.007,1.608)$ \\
\hline No & $74(88.1 \%)$ & $10(11.9 \%)$ & 1 & 1 \\
\hline \multicolumn{5}{|l|}{ Alcohol drink } \\
\hline Yes & $6(66.7 \%)$ & $3(33.3 \%)$ & $5.87(1.39,24.79$ & $0.019(0.001,1.28)$ \\
\hline No & $317(92.2 \%)$ & $27(7.8 \%)$ & 1 & 1 \\
\hline
\end{tabular}

COR: Crude odds ratio, AOR: Adjusted odds ratio, *significantly associated variables at $p<0.05$

\section{Discussion}

The finding of this study showed that relatively low prevalence of STI syndromes were observed among HIV patients. Majority of the patients who had STI syndromes failed treatment with their partners. A range of possible predictors such as socio demographic (Age and marital status) and behavioral (having new sexual partner) were associated with sexually transmitted infections based on syndromes among HIV patients in Ayder hospital, ART clinic.

The overall prevalence of STIs based on the syndromic approach of this study was $30 / 353(8.5 \%)$ with specific syndromic prevalence of 7 patients $(4.6 \%)$ urethral discharge syndrome, 10 patients $(2.8 \%)$ genital ulcer syndrome, 5 patients $(2.5 \%)$ lower abdominal pain syndrome, 1patient $(0.3 \%)$ inguinal bubo and 11 patients $(5.5 \%)$ vaginal discharge syndrome.

Result of this study showed that STI prevalence was low as compared to similar studies conducted previously. A study conducted at Gondar town, showed the overall prevalence $383 / 1071 \quad(35.8 \%)$ and specifically (38.38\%), vaginal discharge, (13.58\%) urethral discharge, LAP (2.87\%), Genital ulcer $(2.09 \%)$ and genital swelling (1.04\%) [30].In Thailand showed the overall prevalence of 526/1,124 (47.0\%) [31]. and in urban Peru showed a prevalence of $(27 \%)$ and
$42 \%$ genital discharge, $7.2 \%$ genital ulcer [28].

This low prevalence could be due to; small sample size, time of study ( awareness of individual patients about prevention measures from gettingSTIs could be changed through time, and because of the general poor health seeking behavior of the community. The level of awareness to distinguish between abnormal vaginal discharges from the normal ones, in women, could be one factor.

The prevalence of STIs of this study was relatively higher as compared to the study conducted in Adamitulu woreda which was $(3.3 \%)$ [38].This may be due to; the study conducted in Adamitulu woreda was community based and it was not specific to risk groups like the current study [15].

Recurrent cases in this study were reported $26.7 \%(8 / 30)$ of patients with STIs, $93.3 \%(28 / 30)$ STI patients failed to bring sexual partners to be treated and 33.3\%(10/30) STI patients did not use condom consistently. It was relatively high, when compared to the result of similar study conducted in Gondar town (no partner treatment $77 \%$, recurrent cases $2.1 \%$ ). This may indicate that the health service providers (ART staff) did not fully council and convince for the patients on the components of STI syndrome management flow chart during every follow up visit, or the patients did not accept what they were counseled and educated at each follow up visit by the health care provider. Treatment failure due to several other 
factors could be the reason for the recurrence like, failure to bring sexual partner, inconsistent use of condom, antimicrobial resistance, poor compliance to treatment, HIV co-infection itself, and another opportunistic infections.

Age, marital status, having new sexual partner within the last three months were the factors significantly associated with STIs based on the syndromes in this study.

The odds of having STIs were 11.4 times higher among HIV patients with the age range between 15-34, when compared with the age group above or equal to $35[\mathrm{AOR}=11.39(95 \% \mathrm{CI}=1.1,116.5)]$. This was in line with the findings of similar study conducted at Gondar town [29], Thailand [27], India [34] and a study conducted among pregnant women in Zimbabwe [37].The reasons why the younger HIV patients were at high risk of getting STIs than the elder once might be due to; they are sexually active group and their awareness on safer sexual behavior might be lower. Peer pressure, may be due to they had multiple sexual partners because, most of them were unmarried. In contrast to the above, being older (greater than 30 years of age) were risks for herpes simplex virus type 2 (HSV-2) in a similar study conducted among drug abusers [35].Even though the risk age groups to have HSV-2 infection are from 15-25years, but mostly they will not be symptomatic, if their cell mediated immunity is intact, so as age increases immunity may be decreased, as a result the latent infection will be reactivated and become symptomatic.

The odds of having STIs among married HIV patients were $96.9 \%$ at reduced risk, when compared with single HIV patients $\quad[\mathrm{AOR}=0.031(95 \% \mathrm{CI}=0.001,0.934)]$. This was consistent with similar study conducted in Bangladesh [38]. Singe HIV patients were at high risk of getting STIs than married HIV patients may be due to, they had multiple sexual partners, may be relatively younger (sexually active), high chance to have sex with commercial sex workers. But this finding was inconsistent study conducted at Gondar town [28], Thailand [27], India [34] and Zimbabwe [37]. In these studies marital status were not a factor may be due to; the study participants were out layered to one side(single or married group).may be due to some cultures did not allow sexual intercourse before marriage.

The odds of having STIs were 152 times higher among HIV patients having new sexual partner within the last three months than the odds of STIs without new sexual partner $[\mathrm{AOR}=152(3.7,6224)]$ this was in line with the study conducted in Addis Ababa(p value $=0.01, \mathrm{CI}=0.018$ to 0.65 ) [32]. A study conducted among young Ghanaian women [36].Similarly a study conducted among pregnant women in Zimbabwe $\quad(\mathrm{AOR}=1.16 ; 95 \% \mathrm{CI}$ : 1.01-1.33) [37].This happens because of the probability of having STIs is increased with having new or multiple sexual partners.

\section{Conclusion}

The prevalence of sexually transmitted infections based on the syndromic approach among HIV patients was $8.5 \%$ and the factors associated with STIs among HIV patients were: age, marital status, having new sexual partner within the last three months.

\section{Recommendation}

Regional health bureau

- Woreda to region-wide awareness creation on the safer sexual behaviors, impact of multiple sexual partnerships, modes of transmission, signs and symptoms of the different types of syndromes of STIs, specifically for young age groups (15-34 years) and un married sexually active individuals.

For all Health professionals

- Should take time to council and convince on the components of the national STI syndromic management flow chart during every follow up.

- Increasing awareness of premarital test for STIs and encouraging couples to use the service.

- Moreover, further studies to explore the predictor variables are highly recommended.

\section{Acknowledgments}

We are thankful to all respondents for their willing to participate in the study as it is through their cooperation that we have been able to write this report.

We also would like to thank to Mekelle University for the financial support. Our appreciation also extends to data collectors and Ayder referral hospital ART clinic staff for diligent efforts and hospitality.

\section{References}

[1] Schryer AD, Meheus A. Epidemiology of sexually transmitted disease: the global picture. Bull. WHO 1990; 68:639-654.

[2] WHO. Global Prevalence and Incidence of Selected Curable Sexually Transmitteddiseases. Summary Sheets.WHO, Department of HIV/AIDS, Geneva, 2001.

[3] GutheT. Venereal diseases in Ethiopia. Bull WHO $1949 ; 2: 85-137$

[4] Kloos H, Zein ZA. Health and Disease in Ethiopia: A Guide to the Literature 1940-1985. Ministry of Health, Addis Ababa, 1988

[5] Molla M, Ismail S, KumieA et al. Sexual violence among female street adolescents in Addis Ababa, April 2000. Ethiop J Health Dev 2002; 16:119-128.

[6] Wanzahun Godana, Abraham Atta. Prevalence of HIV/AIDS and its Associated Factors among Prevention of Mother-to-Child Transmission (PMTCT) Service Users in Jinka Town Health Institutions, South Omo Zone, South Ethiopia. Science Journal of Public Health. Vol. 1, No. 3, 2013, pp. $125-130$.

[7] Fleming DT, Wasserheit JN: From epidemiological synergy to public health policy and practice: the contribution of other sexually transmitted diseases to sexual transmission of HIV infection.SexTransm Infect 1999, 75(1):3-17. 
[8] Centers for Disease Control and Prevention (CDC): Incorporating HIV prevention into the medical $\mathrm{c}$ of persons living with HIV. Recommendations of CDC, the Health Resources and Services Administration, the National Institutes of Health, and the HIV Medicine Association of the Infection Diseases Society of America. MMWR Recomm Rep 2003, 52(RR-12):1-24.

[9] World Health Organization: HIV/AIDS program: strengthening health services to fight HIV/AIDS. Essential prevention and care interventions for adults and adolescents living with HIV in resource-limite settings. Geneva: WHO press; 2008.

[10] Meheus AZ. Practical approaches in developing nations. In: Holmes KK, Mardh P-A, Sparling PF, et al , eds. Sexually transmitted diseases. 1st ed. New York: McGraw-Hill, 1984:998-1008.

[11] World Health Organization. Guidelines for the management of sexually transmitted infections. Geneva: WHO/HIV AIDS/2001, 01 .

[12] Lush L, Walt G, Ogden J. Transferring policies for treating sexually transmitted infections: what's wrong with global Guidelines? Health Policy and Planning2003; 18:18-30.

[13] Wolday D. Prevalent infectious diseases in patients with HIV/AIDS in Ethiopia. Ethiop Med J 2003; 41:189-203.

[14] Nazish Fatima et al.: Sero Prevalence of Syphilis Infection among Patients Attending Antenatal Care \& Sexually Transmitted Disease (STD) Clinics: Observations from a Tertiary Care Hospital of Northern India. American Journal of Internal Medicine 2014; 2(1): 6-9

[15] Wolday D, Gebre-Mariam Z, Mohamed Z et al. Risk factors associated with failure of syndromic treatment of sexually transmitted diseases among women seeking primary care in Addis Ababa. Sex Transm Infect 2004;80:392-394

[16] Kebede Y, Dorigo-Zetsma W, Mengistu Y et al. Transmission of herpes simplex virus Type 2 among factory workers in Ethiopia. J Infect Dis 2004; 190:365-372.

[17] AIDS Control and Prevention (AIDSCAP) Project/Family Health International. Control of Sexually Transmitted Diseases: A Handbook for the Design and Management of Programs. Addis Ababa.

[18] WHO: Global prevalence and incidence of selected curable sexually transmitted Diseases, overview and estimates. Geneva: WHO; 2001.

[19] Nuwaha F: Determinants of choosing public or private health care among patients with sexually transmitted infections in Uganda. Sex Transmitted Dis 2006, 33:422-427.

[20] Glasier A, Gülmezoglu AM, Schmid GP, Moreno CG, Van Look PF: Sexual and Reproductive health, a matter of life and death. Lancet 2006, 368:1595-1607.

[21] Rizvi H., ZuberiF.Women's health in developing countries.Obstet.Gynecol. 2006; 20: 907- 922.

[22] Menendez C., Castellsague X., Renom M et al. Prevalence and risk factors of sexually transmitted infections and cervical neoplasiain women from a rural area of southern Mozambique.
Infec. Dis. Obst. Gyn. Article ID 2010; 609315: 1-9.

[23] Moodley P., Sturm A. Management of vaginal discharge syndrome: how effective is our strategy. Inter. Jour. Antimi. Agents. 2004; 24S: S4-S7.

[24] Mullick S., Watson-Jones D., Beksinska M., Mabey D. Sexually transmitted infections in pregnancy: prevalence, impact on pregnancy outcomes and approach to treatment in developingcountries.Sex.Transm. Infect. 2005; 81:294-302

[25] Ros C., Schmitt C. Global epidemiology of sexually transmitted diseases.AsianJ.Androl. 2008; 10: 110-114.

[26] Mullick S., Watson-Jones D., Beksinska M., Mabey D. Sexually transmitted infections in pregnancy: prevalence, impact on pregnancy outcomes and approach to treatment in developing countries.Sex.Transm. Infect. 2005; 81: 294-302

[27] SuvannaA, Rangisma L, Anuvat R, et al. sexually transmitted infection among HIV infected women in Thailand, 2012.

[28] Clark JL, Lescano AG, Konda KA, Leon SR, Jones FR, et al. Syndromic Management and STI Control in Urban Peru. PLoS ONE 2009; 4(9): 1.

[29] PlordeD. Sexually transmitted diseases in Ethiopia: Social factors contributing to their spread and implications for developing countries. Br. J. Vener. Dis. 1981; 57: 357-362.

[30] http://www.biomedcentral.com/1471-2458/13/143[Accessed on may15th , 2014]

[31] Ademe M, Syndromic management approach and lab. Diagnosis of T.vaginalis in STI compliant in Merawi health center, 2011

[32] Kassa A, Shume A and Kloos H. Sexually Transmitted Infections. In: Berhane Y, Hailemariam D and Kloos (Eds): The Epidemiology and Ecology of Health and Disease in Ethiopia. Shama Books, Addis Ababa, Ethiopia.2006: 435-45.

[33] Tesfaye F, Kasseye M, Kebede D et al. Community-based survey of STI syndromes in Adami-Tulu. Ethiop J Health Dev 2000;14:7-12.

[34] Marg R. STD and HIV Prevalence Survey among Female Sex Workers and Truckers on Highway Routes in the Terai, Nepal. Kathmandu, Nepal Family Health International/Nepal Bakundol, PulchowkLalitpur, Nepal 2000.

[35] Hwang L-Y, Ross MW, Zack C, Bull L, Rickman K, Holliman M. Prevalence of Sexually Transmitted Infections and Associated Risk Factors among Populations of Drug Abusers. Clinical Infectious Diseases 2000;31:920-6.

[36] S.A. Ohen IOA. Factors associated with sexually transmitted infections among young Ghanaian women. Ghana medical journal 2008;42(3):96-100.

[37] N. E. Kurewa MPM, M. W. Munjoma et al. The burden and risk factors of Sexually Transmitted Infections and Reproductive Tract Infections among pregnant women in Zimbabwe. BMC Infectious Diseases2010; 10::127.

[38] Shahidullah MMRM. Adolescent Self-Reported Reproductive Morbidity and Health Care Seeking Behavior in Bangladesh Paper prepared for 25 th IUSSP International Population Conference2005 18-23. 